\title{
Albendazole Solid Dispersions: Influence of Dissolution Medium Composition on In Vitro Drug Release
}

\author{
Noelia L. Gonzalez Vidal ${ }^{1,2}$, Silvina G. Castro ${ }^{3}$, \\ Sergio F. Sanchez Bruni ${ }^{4}$, Daniel A. Allemandi ${ }^{3}$, \\ and Santiago D. Palma ${ }^{3, *}$ \\ 'Cátedra Control de Calidad de Medicamentos, Departamento de Biología, Bioquímica y Farmacia, Universidad Nacional del Sur, \\ San Juan 670, B8000ICN Bahía Blanca, Argentina \\ ${ }^{2}$ Consejo Nacional de Investigaciones Científicas y Technológicas, San Jaun 670, Bahia Blanca, Argentina \\ ${ }^{3}$ Departamento de Farmacia, Facultad de Ciencias Químicas, Universidad Nacional de Córdoba, UNITEFA-CONICET, \\ Ciudad Universitaria, X5000HUA Córdoba, Argentina \\ ${ }^{4}$ Laboratorio de Farmacología, Departamento de Fisiopatología, Facultad de Ciencias Veterinarias. \\ Universidad Nacional del Centro de la Pcia. de Buenos Aires. Campus Universitario, 7000 Tandil, Buenos Aires, Argentina
}

\begin{abstract}
The rate-limiting step to drug absorption is often dissolution from the dosage form, especially for poorly soluble compounds. Two possibilities for improving the dissolution of these drugs are to increase the available surface area and to improve their apparent solubilities under physiologically relevant conditions with surfactants as wetting agents. Albendazole (ABZ), one of the most effective broad-spectrum antihelminthic agents, has a very low aqueous solubility, which leads to an erratic availability. Solid dispersions (SD) with different amounts of carriers (P188 and PEG6000) were formulated to improve the ABZ dissolution rate. When the dissolution test is used to infer biopharmaceutical properties of the dosage form, it is essential that the method simulates the gastrointestinal conditions. The objective of this study was to examine the applicability of different dissolution media to the evaluation of ABZ and ABZ-SD dissolution rates. Dissolution profiles were performed by the official method $(0.1 \mathrm{~N} \mathrm{HCl})$ and Simulated Gastric Fluid modified with a surfactant. Wetting was evaluated through the determination of surface tension and contact angle of the solutions. The dissolution rate of ABZ was clearly affected by the variables assessed in this study. These results have implications in the design of physiologically based dissolution media.
\end{abstract}

KEYWORDS: Albendazole; contact angle; surface tension; dissolution rate; solid dispersions.

\section{INTRODUCTION}

$\mathrm{C}^{\mathrm{r}}$ issolution testing is used to assess drug release rate from a dosage form, and it may provide a rational basis to predict in vivo behavior and dosage form optimization (1). The selection of the dissolution medium is based, in part, on drug solubility data and product dose range to ensure that sink conditions are met. On the other hand, when the dissolution test is used to infer the biopharmaceutical properties of the dosage form, a key experimental condition is that the proposed method simulates the gastrointestinal environment as much as possible (2). In spite of this, compendial dissolution media are usually far from representative of gastrointestinal tract conditions (3). The development of the Biopharmaceutics Classification System (BCS) has renewed interest in the compounding of physiologically relevant media for dissolution testing $(4,5)$. The lack of similarity between compendial methods for in vitro dissolution testing and physiologic conditions is reported as the main reason for the inability to predict in vivo dissolution from in vitro data (3). So, it would be highly desirable to develop media that more adequately reflect

${ }^{*}$ Corresponding author. physiological conditions (6). In addition to $\mathrm{pH}$, an important characteristic of gastric fluids is the lower surface tension relative to water. Finholt et al. (7) examined the surface tension of human gastric juice and concluded that the value is nearly independent of $\mathrm{pH}$ and secretion rate, lying normally in the range of $35-45 \mathrm{mN} / \mathrm{m}$, while the surface tension of water is $72.8 \mathrm{mN} / \mathrm{m}$. On the other hand, Luner et al. (8) studied the wetting properties of dispersions composed of dominant surface-active species found in the stomach at physiologically relevant concentrations, taking into account that in the fasted state, concentrations of bile salts in the gastric fluid, presumably present due to reflux from the duodenum, are in the range of $0.01-0.08 \mathrm{mM}$. In this sense, the authors studied different simulated gastric fluids and concluded that a given surfactant used to produce wetting similar to in vivo systems may indeed show characteristically different behavior for a particular surface. Consequently, when selecting a surfactant to emulate the properties of gastric fluids for a particular surface, it may be useful to compare the wetting effect of a surfactant-based dissolution medium to that of media simulating in vivo conditions to ensure that a reasonable correspondence in wetting properties is achieved. 
The main possibilities for improving the dissolution of poorly soluble drugs are:

- Increase the available surface area by decreasing the solid particle size, by optimizing the wetting characteristics of the compound surface, or both.

- Decrease the boundary layer thickness.

- Ensure sink conditions for dissolution.

- Improve the apparent solubility of the drug under physiologically relevant conditions (9).

In the 1990s, the Food and Drug Administration (FDA) promoted the use of surfactants in dissolution media for poorly soluble drugs (8). It has been suggested that for poorly soluble compounds, inclusion of synthetic or biological surfactants in dissolution media is important to simulate a biological environment. Thus, in vitro dissolution would be more representative of in vivo rates $(3,10,11)$.

The mechanism by which a surfactant increases the dissolution rate is complex. Surfactants generally improve wetting by reducing the interfacial energy for the liquid-vapor interface and adsorbing on the substrate to cause a reduction in the interfacial energy for the solid-liquid interface. However, some authors (12-15) have demonstrated a solubilizing effect at concentrations below the critical micellar concentration (CMC). A better understanding of the wetting properties of biorelevant media is necessary to rationally formulate dissolution media that accurately account for the critical factors involved in the dissolution process (8).

The drug under investigation in this study was albendazole (ABZ), a low aqueous solubility $(0.01 \mathrm{mg} / \mathrm{mL}$ in water at $25^{\circ} \mathrm{C}$ ) and high permeability antihelminthic agent that belongs to BCS Class 2 (16). The unsatisfactory $A B Z$ dissolution rate limits its absorption; therefore, it is poorly and erratically absorbed from the gastrointestinal tract. In vivo studies $(17,18)$ have demonstrated that the oral absorption of benzimidazole compounds depends on their dissolution at low $\mathrm{pH}$ and is closely related to the rate of gastric emptying and gut transit time. The USP indicates that the dissolution medium used to evaluate $A B Z$ dissolution is $0.1 \mathrm{~N} \mathrm{HCl}$ (19). However, this does not reflect the most relevant physiological conditions of the gastrointestinal tract.

The improvement of dissolution rates may be addressed by mean of different technological strategies. Among them, the design of solid dispersions (SD) has acquired notable attention (20-22). An SD is defined as the dispersion of one or more active ingredients in inert carriers in the solid state prepared by fusion, solvent, or solvent-fusion methods (23).

Misleading results can be concluded from in vitro release rates of SDs if the test conditions do not adequately simulate the gastrointestinal conditions (9).

In this framework, the aim of this work was to evaluate the effect of interfacial properties of different dissolution media on the dissolution rate of $A B Z$ and SDs containing $A B Z$.

\section{MATERIALS AND METHODS}

\section{Preparation of Solid Dispersions}

ABZ (pharmaceutical grade, Parafarm, Buenos Aires, Argentina), Poloxamer 188 (P188, BASF, Germany), and Polyethylene Glycol 6000 (PEG6000, Fluka, Germany) were used for the preparation of SDs.

SDs were prepared by melting different proportions of $A B Z$ and carrier (Table 1 ) in a heating circulator water bath at $63^{\circ} \mathrm{C}$ for P188 and $68^{\circ} \mathrm{C}$ for PEG6000. The mixtures were homogenized by stirring, cooled, and pulverized. The 212- $\mu \mathrm{m}$ particle size fraction was obtained by sieving and kept in a screw-capped glass vial at $2-8{ }^{\circ} \mathrm{C}$ until use.

\section{XRP Diffraction and IR Spectroscopy}

Powder X-ray diffraction was performed using a Rigaku Miniflex 2000 diffractomer ( $\lambda$ : $1.5418 \AA ̊$ with a Bragg-Brentano geometry). The radiation was generated by a Cu K lamp. The instrument was operated in the continuous scan mode with the scanning speed at $2 \% \mathrm{~min}$. The scan range was $3-70^{\circ} 2 \theta / \theta$ with a scan speed $0.066^{\circ} 2 \theta / \mathrm{s}$. The solid dispersions and their respective physical mixtures were also characterized by IR spectroscopy (FT; Nicolet 5SXC FTIR Spectrometer) using KBr disks.

\section{Determination of Surface Tension}

Surface tension measurements were conducted using the du Noüy method. Calibration was performed prior to use of the instrument and then verified using distilled water. The solutions were maintained at $37.0 \pm 0.3^{\circ} \mathrm{C}$ using a heating circulator water bath.

Three separate measurements were made on separate aliquots of each of the following solutions: (1) $0.1 \mathrm{~N}$ hydrochloric acid; (2) modified simulated gastric fluid (mSGF) pH 1.2 without pepsin, with $0.25 \% \mathrm{w} / \mathrm{v}$ sodium lauryl sulfate (24); and (3) solutions of both media with different concentrations $\left(0.65,1.95,5.82\right.$, and $\left.12.1 \times 10^{-5} \mathrm{M}\right)$ of each carrier. A wide range was selected to evaluate the influence of the carrier concentration on the dissolution media.

\section{Contact Angle Determination}

A rough estimation of hydrophobic drug wettability could be obtained by liquid-solid interface contact angle measurement (3). This assay was performed on compacted powders ( $A B Z$, carriers, and SD) using a homemade goniometer equipped with an image analysis attachment. The wetting liquid was contained in a $50-\mu \mathrm{L}$ micropipette

TABLE 1. Composition of Solid Dispersions

\begin{tabular}{ccc}
\hline Solid Dispersion & ABZ $(\% \mathbf{w} / \mathbf{w})$ & Carrier $^{\boldsymbol{a}}(\% \mathbf{w} / \mathbf{w})$ \\
\hline SD1 & 5 & 95 \\
\hline SD2 & 10 & 90 \\
\hline SD3 & 25 & 75 \\
\hline SD4 & 50 & 50 \\
\hline
\end{tabular}

a $P 188$ or PEG600 
(Boeco, Germany); the discharge height was $2.00 \pm 0.02$ $\mathrm{mm}$ and was regulated by a micrometer of $0.01-\mathrm{mm}$ resolution. Parameters such as drop volume, base diameter, and contact angle were recorded as a function of time, using specific software (ImageJ: Drop analysis - DropSnake). The procedure was performed in quadruplicate.

\section{Dissolution Test}

Dissolution tests of powdered SDs containing $50 \mathrm{mg}$ of $A B Z$ were carried out on a suitably calibrated USP Apparatus 2 (Sotax AT7 Smart) at $50 \pm 1 \mathrm{rpm}$, using $900 \mathrm{~mL}$ of dissolution medium at $37.0 \pm 0.5^{\circ} \mathrm{C}$. The dissolution media were $0.1 \mathrm{~N} \mathrm{HCl}$ and $\mathrm{mSGF}(19,24)$. Samples $(5 \mathrm{~mL})$ were withdrawn at predetermined time intervals within one hour, with replacement of the same volume of fresh medium after each withdrawal. Previous results indicated that $A B Z$ incorporated in SDs was rapidly released and reached steady state at 48-72 h (25). The samples were filtered through a nylon membrane disposable filter $(25-\mathrm{mm}$ diameter, 0.45- $\mu$ m pore size, Microclar, Argentine), which was validated for the absence of filter adsorption. Samples were suitably diluted and analyzed using UV spectrophotometry (Thermo Evolution 300) at $297 \mathrm{~nm}\left(\lambda_{\max }\right.$ for $0.1 \mathrm{~N}$ $\mathrm{HCl})$ and $307 \mathrm{~nm}\left(\lambda_{\max }\right.$ for $\left.\mathrm{mSGF}\right)$. The dissolution tests were performed in triplicate. In a previous work (26), the absence of interference on the $A B Z \lambda_{\text {max }}$ in the presence of dissolved carriers was verified. The concentration in each sample was calculated from an ABZ standard calibration curve. Results were averaged, and cumulative drug release percentages were calculated for dissolution profile estimation.

\section{RESULTS AND DISCUSSION}

\section{XRP Diffraction and IR Spectroscopy}

XRD diffractograms for the systems ABZ/P188 and ABZ/ PEG6000 are shown in Figure 1. Some signals allowed us to identify each component, $A B Z(\theta / 2 \theta): 6.96,11.37,17.91$, 22.12, 24.58; P188 ( $\theta / 2 \theta)$ : 19.32, 23.46; and PEG6000 $(\theta / 2 \theta)$ : $19.20,23.50$.

Diffractograms from SDs clearly showed that the signals assigned to each component were practically unchanged. Thus, no interactions between them were detected, although SDs evidenced a slight reduction in cristallinity, especially at a high drug/carrier ratio (SD1).

The ABZ spectrum shows an $\mathrm{N}-\mathrm{H}$ stretching vibration at 3,324.94 $\mathrm{cm}^{-1}$ and a bending vibration at 1,653.07 $\mathrm{cm}^{-1}$ (Figure 2). The signals at these wavenumbers remain unaltered for SDs. In this way, the infrared spectra confirm that there were no chemical interactions between $A B Z$ and carriers (P188 or PEG6000) because of their close contact in SDs.

\section{Determination of Surface Tension}

The surface tension obtained for $0.1 \mathrm{~N} \mathrm{HCl}$ was 54.29 $\mathrm{mJ} / \mathrm{m}$, while for $\mathrm{mSGF}$ was $34.30 \mathrm{~mJ} / \mathrm{m}$, similar to that of gastrointestinal fluid (24). The surface tension was also measured at increasing concentrations of carrier (Figures 3 and 4). For mSGF, surface tension was not affected by

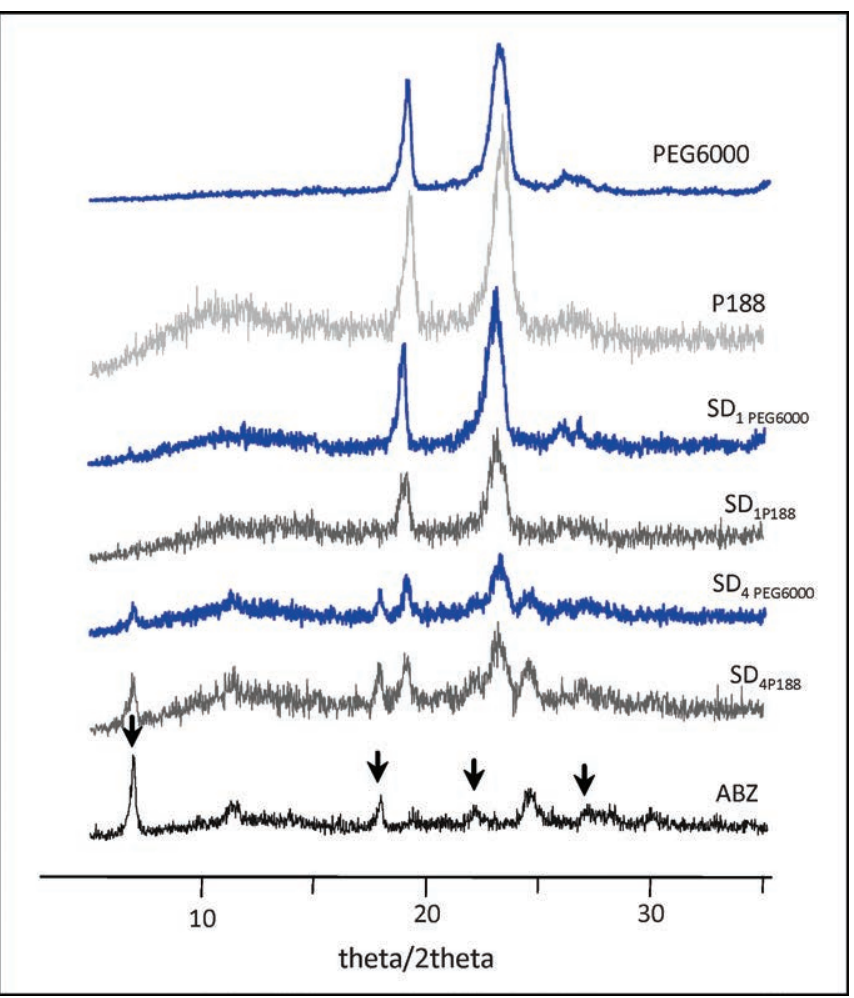

Figure 1. X-ray diffractograms of ABZ, P188, PEG6000, and SDs.

A

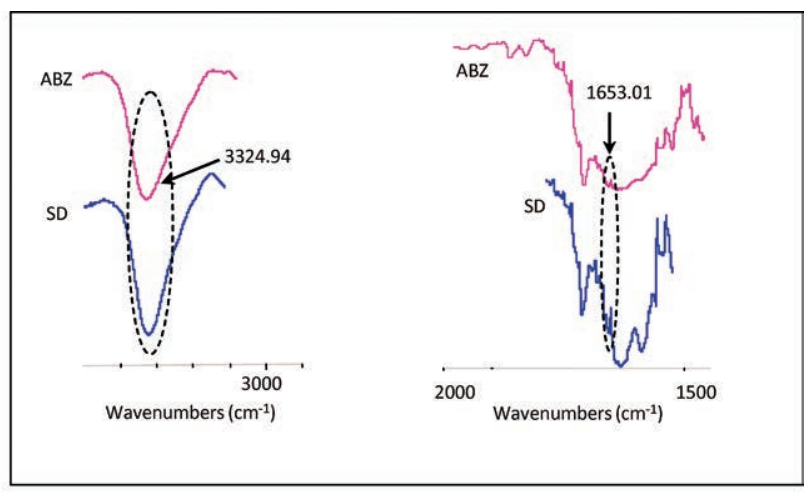

B
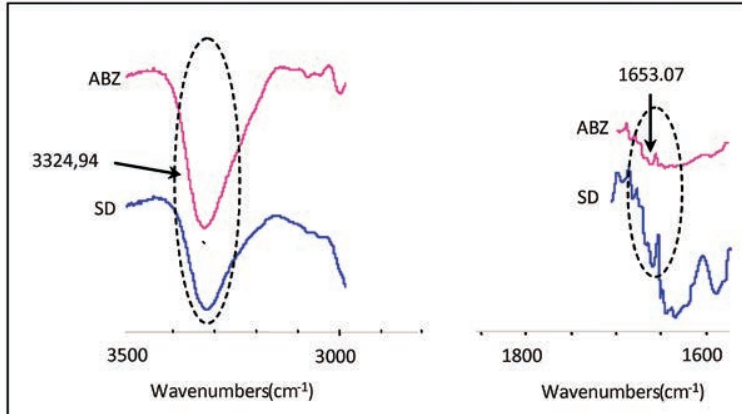

Figure 2. IR spectra of ABZ and SDs in (A) PEG6000 and (B) P188. 
the presence of PEG6000 or P188. On the other hand, for $0.1 \mathrm{~N} \mathrm{HCl}$, the surface tension increased incrementally at increasing concentrations of PEG6000 until $1 \times 10^{-5} \mathrm{M}$, followed by a decrease (Figure 3). This effect was studied by Hillgren and Aldén (27), who described PEG as a nonionic water-soluble polymer with poor surface activity at low concentrations. However, at higher concentration, the surface tension begins to decrease. This effect was attributed to the $\mathrm{CH}_{2} \mathrm{CH}_{2}$ groups, which are sufficiently hydrophobic to form an adsorbed layer from a PEG solution and organize a spreading monolayer at the air-water interface (28). It is important to note that the surface tension for $0.1 \mathrm{~N}$ $\mathrm{HCl}$ with PEG6000 was greater than for mSGF.

On the other hand, the surface tension decreases at increasing concentrations of P188 in $0.1 \mathrm{~N} \mathrm{HCl}$ (CMC $1.25 \times 10^{-4}$ $\mathrm{M})$ due to the surfactant properties of this carrier (29). Never-

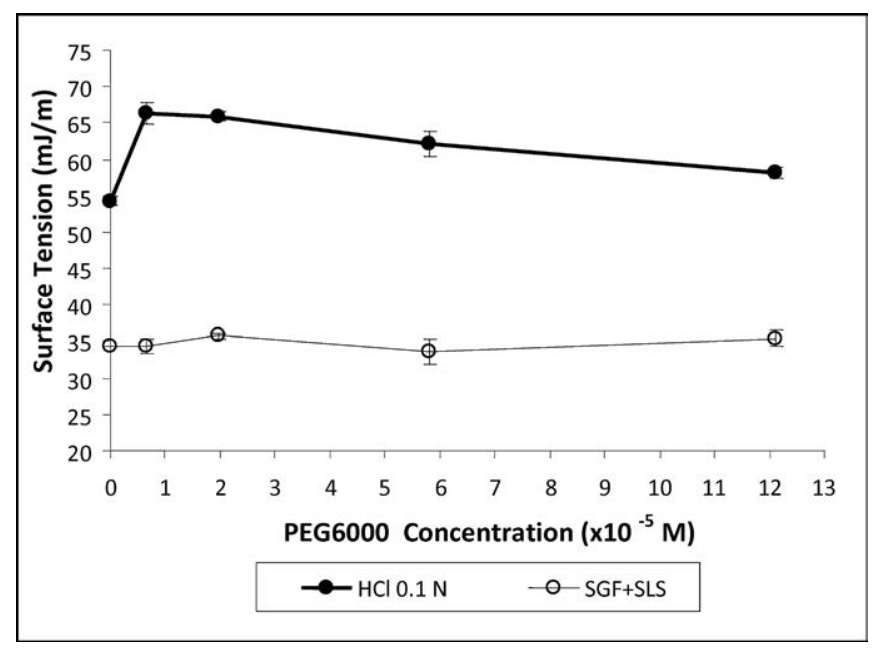

Figure 3. Surface tension of different dissolution media at increasing PEG6000 concentrations.

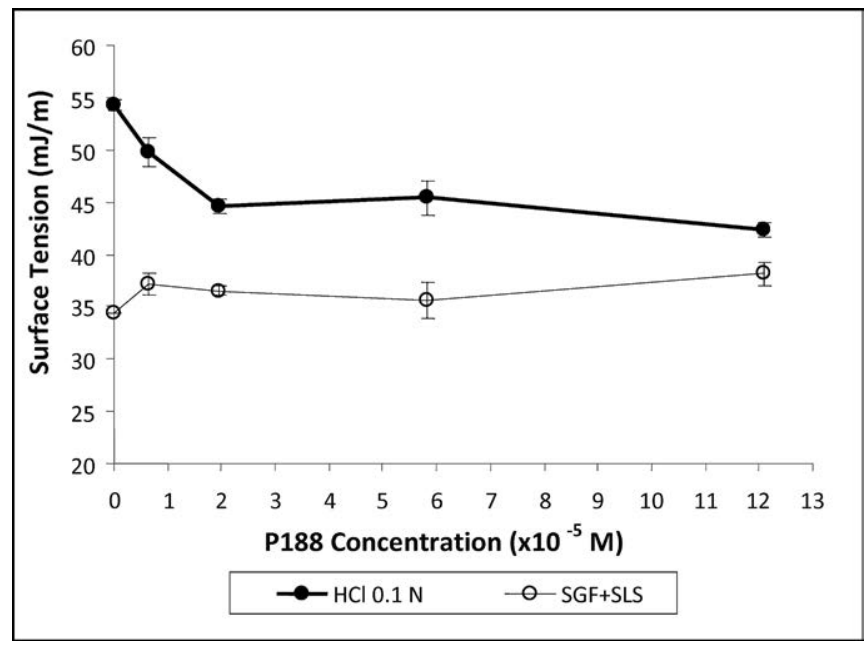

Figure 4. Surface tension of different dissolution media at increasing P188 concentrations. theless, the surface tension values were still higher for $0.1 \mathrm{~N}$ $\mathrm{HCl}$ than for mSGF in the presence of P188 (Figure 4).

\section{Contact Angle Determination}

ABZ showed very low wettability $\left(\alpha \approx 90^{\circ}\right)$, which increased significantly when the drug was incorporated in SDs due to the presence of the carrier (Figure 5).

It is expected that this improvement in water affinity leads to a faster drug dissolution, especially at the earlier stage of the dissolution process. This behavior may be attributed to the change in the hydrodynamic environment of the media produced by the presence of the hydrophilic polymer, which is able to reduce the diffusion layer thickness (30).

\section{Dissolution Tests}

The ABZ dissolution performance was somewhat improved with the use of $\mathrm{mSGF}$, compared with the dissolution rate obtained in $0.1 \mathrm{~N} \mathrm{HCl}$ (Figure 6). Moreover, the $A B Z$ dissolution was also improved with the formulation of SDs, especially with P188 as carrier where the smallest contact angle was observed. This phenomenon can be attributed to an improvement of ABZ particle wettability, regardless of the presence of carrier. When an SD was formulated with PEG6000, the dissolution was particularly improved for SD1 and SD2 (Figure 6).

The SDs dissolution profiles in both dissolution media are shown in Figure 6. The results show that the dissolution was clearly different in both media, which confirms the primary hypothesis of this work. The dissolution rate of SDs with P188 as carrier was higher in mSGF than in $0.1 \mathrm{~N} \mathrm{HCl}$ in almost all cases. This is due to the presence of a surfactant, which reduced the surface tension, promoting the particle wettability, and therefore its dissolution. A similar behavior was observed for SDs prepared with PEG6000, at lower ABZ concentrations and higher carrier concentrations (SD1 and SD2), due to the dependence of the dissolution rate on carrier concentration.

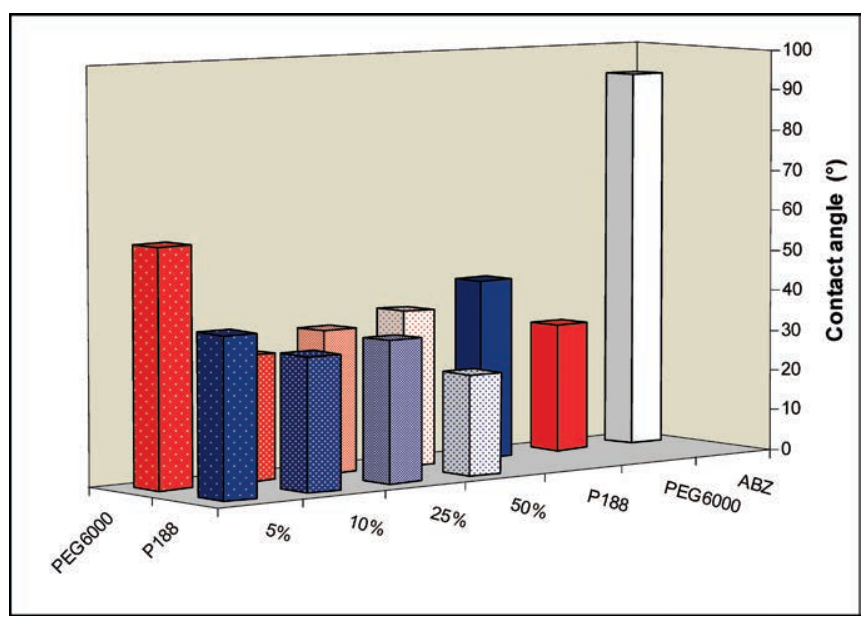

Figure 5. Determination of contact angle of $A B Z$, carriers, and SDs (PEG6000 or P188 with increasing concentrations of $A B Z$ ). 

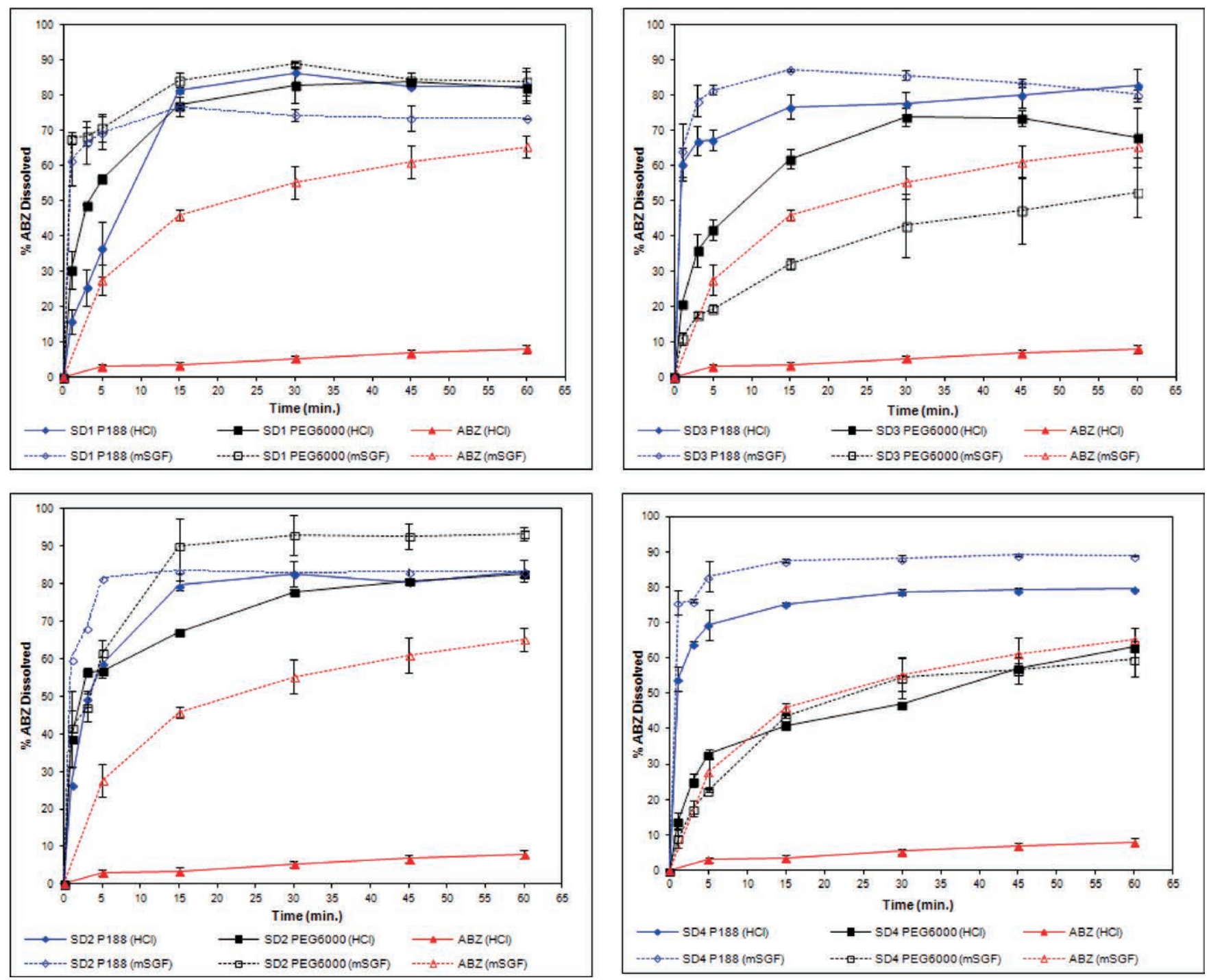

Figure 6. Dissolution profiles of $A B Z$ and SDs in both dissolution media.

\section{CONCLUSION}

The present study reveals that ABZ dissolution characteristics vary widely depending on the dissolution media. This can be attributed to a lower surface tension of the alternative dissolution medium (i.e., with SLS), which favors the wettability of the drug and, therefore, improves the $A B Z$ dissolution rate. This kind of information may be useful in the proposal of official monographs for dissolution assays that resemble physiological conditions. On the other hand, SDs containing ABZ and P188 or PEG6000 as carriers showed a significant increase in drug dissolution rate, which confirms the suitability of this technology for dissolution improvement of a Class 2 active ingredient.

\section{CONFLICT OF INTEREST}

The authors report no conflict of interest.

\section{REFERENCES}

1. Dissolution Testing of Immediate Release Solid Oral Dosage Forms; Guidance for Industry; U.S. Department of Health and Human Services, Food and Drug Administration, Center for Drug Evaluation and Research (CDER), U.S. Government Printing Office: Washington, DC, 1997.

2. Cohen, J. L.; Hubert, B. B.; Leeson, L. J.; Rhodes, C. T.; Robinson, J. R.; Roseman, T. J.; Shefter, E. The Development of USP Dissolution and Drug Release Standards. Pharm. Res. 1990, 7 (10), 983-987. DOI: 10.1023/A:1015922629207.

3. Hörter, D.; Dressman, J. B. Influence of physicochemical properties on dissolution of drugs in the gastrointestinal tract. Adv. Drug Deliv. Rev. 2001, 46 (1-3), 75-87. DOI: 10.1016/S0169-409X(00)00130-7.

4. Amidon, G. L.; Lennernäs, H.; Shah, V. P.; Crison, J. R. A Theoretical Basis for a Biopharmaceutic Drug 
Classification: The Correlation of in Vitro Drug Product Dissolution and in Vivo Bioavailability. Pharm. Res.

1995, 12 (3), 413-420. DOI: 10.1023/A:1016212804288.

5. Galia, E.; Nicolaides, E.; Hörter, D.; Löbenberg, R.;

Reppas, C.; Dressman, J. B. Evaluation of various dissolution media for predicting in vivo performance of class I and II drugs. Pharm. Res. 1998, 15 (5), 698-705. DOI: 10.1023/A:1011910801212.

6. Yu, L. X.; Amidon, G. L.; Polli, J. E.; Zhao, H.; Mehta, M. U.; Conner, D. P.; Shah, V. P.; Lesko, L. J.; Chen, M.-L.; Lee, V. H.; Hussain, A. S. Biopharmaceutics Classification System: The Scientific Basis for Biowaiver Extensions. Pharm. Res. 2002, 19 (7), 921-925. DOI: $10.1023 / A: 1016473601633$.

7. Finholt, P.; Solvang, S. Dissolution kinetics of drugs in human gastric juice - the role of surface tension. J. Pharm. Sci. 1968, 57 (8), 1322-1326. DOI: 10.1002/ jps.2600570809.

8. Luner, P. E.; VanDer Kamp, D. Wetting characteristics of media emulating gastric fluids. Int. J. Pharm. 2001, 212 (1), 81-91. DOI: 10.1016/S0378-5173(00)00602-5.

9. Leuner, C.; Dressman, J. B. Improving drug solubility for oral delivery using solid dispersions. Eur. J. Pharm. Biopharm. 2000, 50 (1), 47-60. DOI: 10.1016/S09396411(00)00076-X.

10. Galia, E.; Hörton, J.; Dressman, J. B. Albendazole Generics-A Comparative In Vitro Study.

Pharm. Res. 1999, 16 (12), 1871-1875.

DOI: 10.1023/A:1018907527253.

11. Luner, P. E. Wetting properties of bile salt solutions and dissolution media. J. Pharm. Sci. 2000, 89 (3), 382-395. DOI: 10.1002/(SICl)1520-6017(200003)89:3<382::AIDJPS9>3.0.CO;2-H.

12. Parfitt, G. D. Fundamental Aspects of Dispersion. In Dispersion of Powders in Liquids, 2nd ed.; Parfitt, G. D., Ed.; Wiley: New York, 1973; pp 1-43.

13. Cid Cárcamo, E. Control de Calidad Biofarmacéutico de Medicamentos. Universidad de Chile: Santiago de Chile, 1992.

14. Wurster, D. E.; Polli, G. P. Investigation of drug release from solids IV. Influence of adsorption on the dissolution rate. J. Pharm. Sci. 1961, 50 (5), 403-406. DOI: 10.1002/jps.2600500506.

15. Wurster, D. E.; Taylor, P. W., Jr. Dissolution kinetics of certain crystalline forms of prednisolone. J. Pharm. Sci. 1965, 54 (5), 670-676. DOI: 10.1002/jps.2600540503.

16. Jung, H.; Medina, L.; Garcia, L.; Fuentes, I.; MorenoEsparza, R. Biopharmaceutics: Absorption Studies of Albendazole and Some Physicochemical Properties of the Drug and Its Metabolite Albendazole Sulphoxide. J. Pharm. Pharmacol. 1998, 50 (1), 43-48. DOI: 10.1111/ j.2042-7158.1998.tb03303.x.

17. Sánchez, S.; Alvarez, L.; Pis, A.; Quiroga, M.; Lanusse, C. Differences in plasma and abomasal kinetics of albendazole and its metabolites in calves grazed on pasture or fed a grain-based diet. Res. Vet. Sci. 1999, 66 (3), 223-230. DOI: 10.1053/rvsc.1998.0264.

18. Lanusse, C. E.; Gascon, L. H.; Prichard, R. K. Gastrointestinal distribution of albendazole metabolites following netobimin administration to cattle: relationship with plasma disposition kinetics. J. Vet. Pharmacol. Ther. 1993, 16 (1), 38-47. DOI: 10.1111/ j.1365-2885.1993.tb00287.x.

19. The United States Pharmacopeia and National Formulary USP 34-NF 29; The United States Pharmacopeial Convention, Inc.: Rockville, MD, 2011.

20. Bley, H.; Fussnegger, B.; Bodmeier, R. Characterization and stability of solid dispersions based on PEG/ polymer blends. Int. J. Pharm. 2010, 390 (2), 165-173. DOI: 10.1016/j.ijpharm.2010.01.039.

21. Wang, X.; de Armas, H. N.; Blaton, N.; Michoel, A.; Van den Mooter, G. Phase characterization of indomethacin in binary solid dispersions with PVP VA64 or Myrj 52. Int. J. Pharm. 2007, 345 (1-2), 95-100. DOI: 10.1016/j. ijpharm.2007.05.046.

22. Sethia, S.; Squillante, E. Solid dispersion of carbamazepine in PVP K30 by conventional solvent evaporation and supercritical methods. Int. J. Pharm. 2004, 272 (1-2), 1-10. DOI: 10.1016/j.ijpharm.2003.11.025.

23. Chiou, W. L.; Riegelman, S. Pharmaceutical applications of solid dispersion systems. J. Pharm. Sci. 1971, 60 (9), 1281-1302. DOI: 10.1002/jps.2600600902.

24. Vertzoni, M.; Dressman, J.; Butler, J.; Hampenstall, J.; Reppas, C. Simulation of fasting gastric conditions and its importance for the in vivo dissolution of lipophilic compounds. Eur. J. Pharm. Biopharm. 2005, 60 (3), 413-417. DOI: 10.1016/j.ejpb.2005.03.002.

25. Castro, S. G.; Sanchez Bruni, S.; Lanusse, C. E.; Allemandi, D. A.; Palma, S. D. Improved Albendazole Dissolution Rate in Pluronic 188 Solid Dispersions. AAPS PharmSciTech 2010, 11 (4), 1518-1525. DOI: 10.1208/s12249-010-9517-6.

26. Castro, S. G.; Allemandi, D. A.; Palma, S. D. Unpublished work, 2011.

27. Hillgren, A.; Aldén, M. A comparison between the protection of LDH during freeze-thawing by PEG 6000 and Brij 35 at low concentrations. Int. J. Pharm. 2002, 244 (1-2), 137-149. DOI: 10.1016/S03785173(02)00322-8.

28. Kim, M. W. Surface activity and property of polyethyleneoxide (PEO) in water. Colloids Surf., $A$ 1997, 128 (1-3), 145-154. DOI: 10.1016/S0927-7757(96)03918-0.

29. Maskarinec, S. A.; Hannig, J.; Lee, R. C.; Lee, K. Y. Direct Observation of Poloxamer 188 Insertion into Lipid Monolayers. Biophys. J. 2002, 82 (3), 1453-1459. DOI: 10.1016/S0006-3495(02)75499-4.

30. Noyes, A. A.; Whitney, W. R. The Rate of Solution of Solid Substances in Their Own Solutions. J. Am. Chem. Soc. 1897, 19 (12), 930-934. DOI: 10.1021/ ja02086a003. 